BENIGN prostate hypertrophy (BPH) is the most common benign tumor in men due to obstruction of the urethra and, finally, uremia. Malondialdehyde (MDA) is a product derived from peroxidation of polyunsaturated fatty acids and related esters. Evaluation of MDA in serum represents a non-invasive biomarker of oxidative stress. Prostate-specific antigen (PSA) is a sensitive marker for prostatic hypertrophy and cancer. We analyzed MDA serum levels to evaluate the oxidative stress in BPH. To this end, 22 BPH patients and 22 healthy donors were enrolled. Data show an increase of MDA level in BPH patients and a positive correlation between PSA and MDA levels. In conclusion, we describe a previously unknown relationship between PSA and MDA as an index of inflammation and oxidative stress in BPH.

Key words: Benign prostate hypertrophy, Malondialdehyde, Prostate-specific antigen

\section{Malondialdehyde in benign prostate hypertrophy: a useful marker?}

\author{
Rosaria Alba Merendino ${ }^{1}$, Francesco Salvo ${ }^{2}$, \\ Antonella Saija ${ }^{3}$, Giuseppe Di Pasquale ${ }^{4}$, \\ Antonio Tomaino ${ }^{3}$, Paola Lucia Minciullo5, \\ Giuseppe Fraccica ${ }^{2}$ and Sebastiano Gangemi, ${ }^{5, C A}$
}

${ }^{1}$ Chair of Immunopathology, Department of Human Pathology, ${ }^{2}$ School and Division of Urology, Department of Human Pathology, ${ }^{3}$ School of Pharmacy, Department Farmaco-Biologico, ${ }^{4}$ Department of Internal Medicine, and ${ }^{5}$ School and Division of Allergy and Clinical Immunology, Department of Human Pathology, University of Messina Medical School, University Hospital 'G. Martino', Via Centonze $\mathrm{n}^{\circ}$ 200, is. 98,98123 Messina, Italy

\footnotetext{
${ }^{\mathrm{CA}}$ Corresponding Author

Tel: +39902212096

Fax: + 39902929953

E-mail: sgangemi@eniware.it
}

The most dramatic organic manifestation of andropause in terms of a decrease in quality of life is benign prostate hypertrophy $(\mathrm{BPH})$, in its ultimate consequences a life-threatening condition due to obstruction of the urethra and, finally, uremia. ${ }^{1}$ The process of prostatic hypertrophy involves all four major cell types in the prostate (smooth muscle cells, fibroblast, acinar and basal epithelial cells) and a variety of hormones, growth factors, and their receptors. ${ }^{2}$ There is much evidence to suggest that benign prostatic hypertrophy is an endocrine disease. ${ }^{2}$ In the adult male, testicular androgens regulate development, growth and functional maintenance of the prostate gland. Androgens regulate prostatespecific antigen (PSA) secretion by acinar epithelial cells. $^{2}$ PSA, a serine protease synthesized by benign and malignant prostatic epithelium, is a sensitive serum marker for prostatic hypertrophy and cancer. In fact, increased PSA levels are often seen in carcinomas of the prostate, but have also been reported in benign inflammatory disorders of the prostate. ${ }^{3}$ Lipid peroxidation is a well-established mechanism of cellular injury in both plants and animals. This process, leading to the production of lipid peroxides and their byproducts, and ultimately to the loss of membrane function and integrity, is widely accepted to be involved in the pathogenesis of several human diseases. MDA is an end-product derived from peroxidation of polyunsaturated fatty acids and related esters. In contrast to free radicals, aldehydes are relatively stable and therefore able to diffuse within or out of the cell and to attack targets distant from the site of original free-radical-initiated events. Furthermore, MDA does not just reflect lipid peroxidation, but is also a byproduct of cyclooxygenase activity in platelets, and persistent platelet activation is a common feature of many clinical syndromes associated with enhanced lipid peroxidation. Thus, measurement of MDA levels in plasma or serum provides a convenient in vivo index of lipid peroxidation and represents a non-invasive biomarker of oxidative stress often clinically employed to investigate radical-mediated physiological and pathological conditions. ${ }^{4}$ In the light of these findings, we analyzed MDA serum levels in order to clarify the oxidative stress in benign prostatic hyperplasia. Moreover, we investigated whether a correlation exists between MDA and PSA serum levels. 
To this end, $22 \mathrm{BPH}$ patients (mean age, 65.8 years; range, 56-79 years), selected on the basis of transrectal ultrasound-guided sextant biopsies of the prostate, were enrolled in this study. Twenty-two healthy donors (mean age, 62.1 years; range, 55-76 years) were also enrolled as a control group. Patients and healthy donors signed an informed consent form. Sera were obtained from peripheral blood allowed to clot at room temperature for $2 \mathrm{~h}$, separated by centrifugation at $200 \times g$ for $15 \mathrm{~min}$ in a 4235 A (ALC Int. S.r.L., Milan, Italy) centrifuge, and stored at $-80^{\circ} \mathrm{C}$ until use. PSA levels were measured with the Hybritech Tandem- $\mathrm{R}$ assay with one kit at one side. The inter-assay and intra-assay coefficients of variation of these assays were $<7 \%$ and $<6 \%$, respectively. Plasma MDA levels were measured by means of a colorimetric assay specific for MDA and carried out using a commercially available kit (Calbiochem-Novabiochem Corporation, La Jolla, CA, USA). This method employs a chromogenic reagent, which reacts with MDA at $45^{\circ} \mathrm{C}$, yielding a stable chromophore with maximal absorbance at $586 \mathrm{~nm}$. Differences in serum MDA levels were assessed by one-way analysis of variance and the Student-Newman-Keults test. Data were expressed as the mean \pm standard deviation. A value $p<0.05$ was considered significant. Correlation between PSA and MDA values were calculated with the Spearman rank test. All data were analyzed using the Stat View SE program (Abacus, Berkeley, CA, USA). The mean serum PSA level was $14.34 \pm 11.85 \mathrm{ng} / \mathrm{ml}$, varying from 3.80 to $57.20 \mathrm{ng} / \mathrm{ml}$. Serum MDA levels of BPH patients were significantly higher than those of healthy donors (HD) $(2.12 \pm 0.44$ versus $0.97 \pm 0.30 \mathrm{nmol} / \mathrm{ml})$. These data represent the first evidence of circulating MDA level increase in BPH patients and confirm the wellknown involvement of oxidative stress in $\mathrm{BPH}$.

Programmed death of cells by apoptosis is a protective mechanism of the organism against accumulation and spread of defective cells. A decreased susceptibility of senescent cells in vitro and in vivo to apoptosis by oxidative stress has been observed. ${ }^{5}$ Highly reactive aldehydes (e.g. 4-hydroxynonenal, MDA), products of the lipid peroxidation, are capable of modifying both DNA and proteins, resulting in mutagenic, genotoxic and cytotoxic events. Therefore, high levels of MDA, such as of other reactive aldehydes, may explain DNA base modifications demonstrated not only in prostate cancer, but also in BPH epithelium. As shown in Fig. 1, analyzed data of our study show that MDA levels strongly correlate with PSA levels in BPH patients $(\mathrm{rho}=0.583 ; p=$ 0.0076). Serum PSA is the most useful parameter for

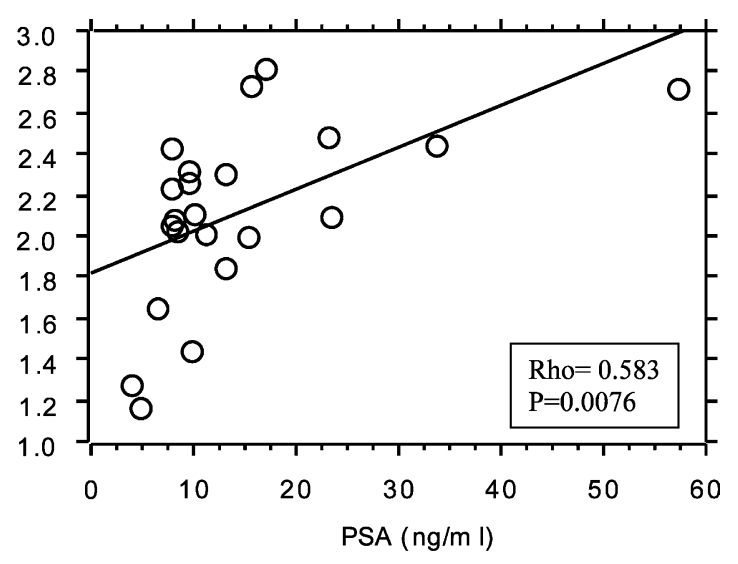

FIG. 1. Correlation between MDA and PSA in BPH.

determining the diagnosis of prostate cancer and the malignant potential of recurrent tumor after radical prostatectomy. Its values are also increased in $\mathrm{BPH}$. Although the high MDA circulating levels observed in $\mathrm{BPH}$ patients strictly demonstrate only the existence of an oxidative stress in this pathological condition, in the light of the strong positive correlation found between PSA and MDA levels in our study, we can speculate that increased circulating MDA levels might be considered a useful marker of lipid peroxidation and inflammation of prostate epithelium. Moreover, the finding of very high MDA levels in a $\mathrm{BPH}$ patient may be considered predictive for prostate cancer. In conclusion, here we describe for the first time a previously unknown relationship between PSA and MDA as an index of inflammation and oxidative stress in $\mathrm{BPH}$ patients. Furthermore, our preliminary findings suggest that an antioxidant such as vitamin $\mathrm{E}$, lycopene or selenium may have a role in preventing prostate cancer progression.

ACKNOWLEDGEMENT. This work was supported in part by a grant from University of Messina Medical School, Italy.

\section{References}

1. Wick G, Janser-Durr P, Berger P, Blasko I, Grubeck-Loebenstein B. Diseases of aging. Vaccine 2000; 18: 1567-1583.

2. Levine AC. Pathogenesis and medical management of benign prostatic hyperplasia. Trends Endocrinol Metab 1995; 6: 128-132.

3. Teillac P, Peiret C, Najean Y, Le Duc A. Prostate-specific antigen in prostatic pathology. Ann Urol (Paris) 1988; 22: 193-196.

4. Meagher EA, FitzGerald GA. Indeces of lipid peroxidation in vivo: strengths and limitation. Free Radic Biol Med 2000; 28: 1745-1750.

5. Muradian K, Schachtschabel DO. The role of apoptosis in aging and agerelated disease: update. $Z$ Gerontol Geriatr 2001; 34: 441-446.

\section{Received 2 January 2003 \\ Accepted 21 January 2003}




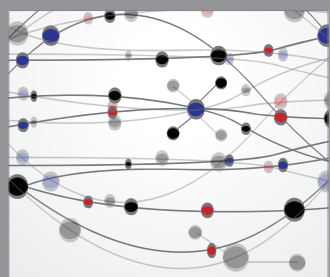

The Scientific World Journal
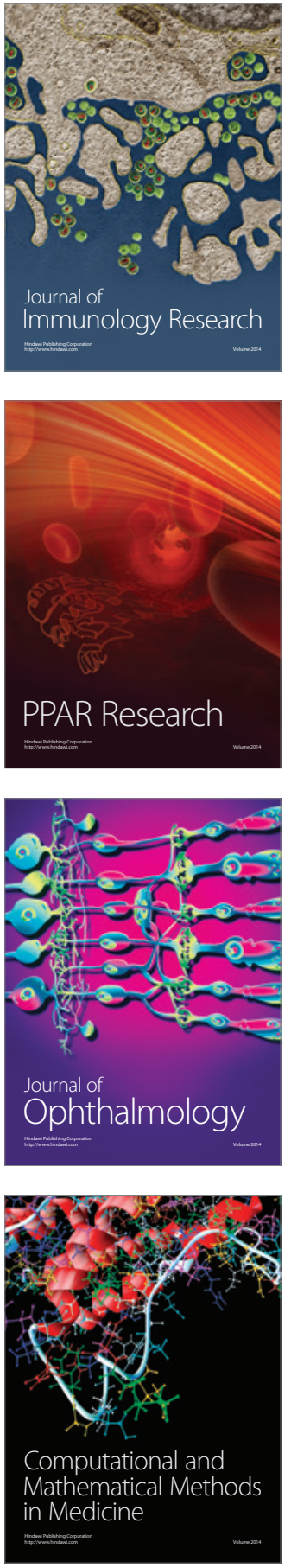

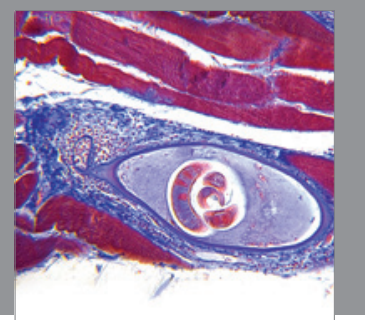

Gastroenterology

Research and Practice
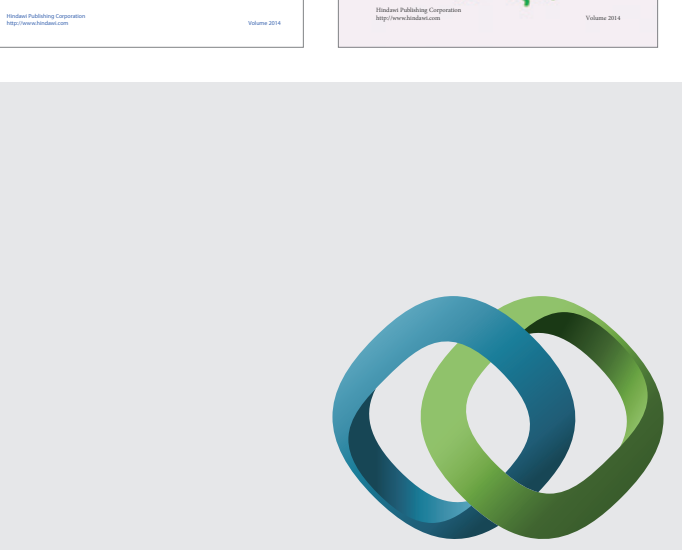

\section{Hindawi}

Submit your manuscripts at

http://www.hindawi.com
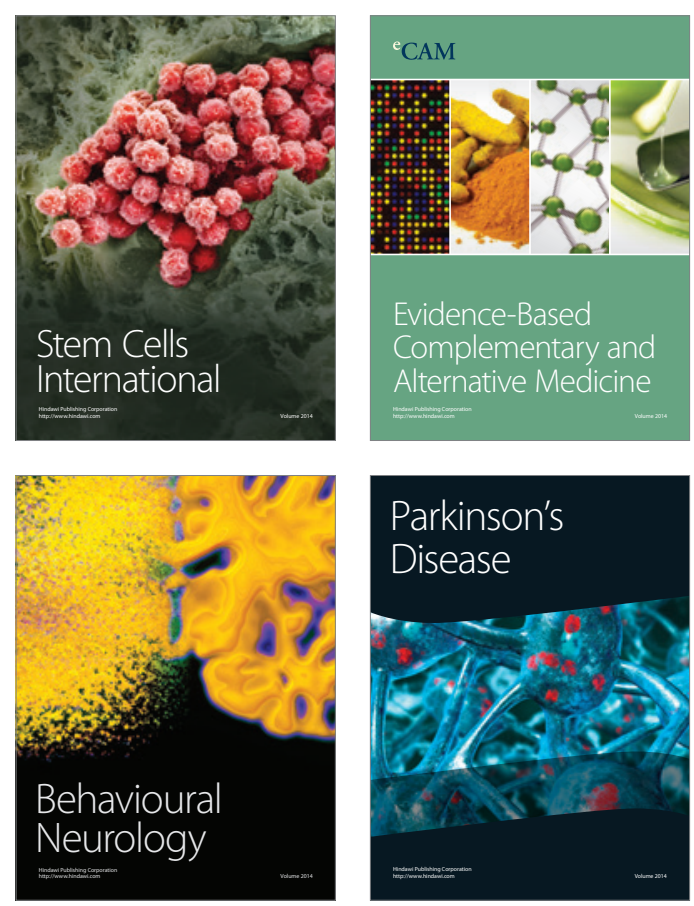

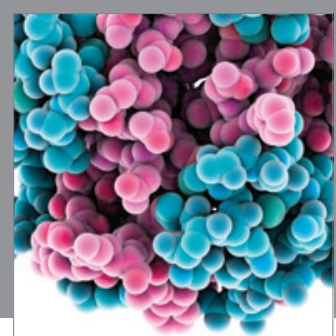

Journal of
Diabetes Research

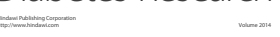

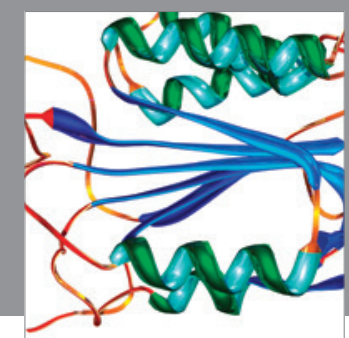

Disease Markers
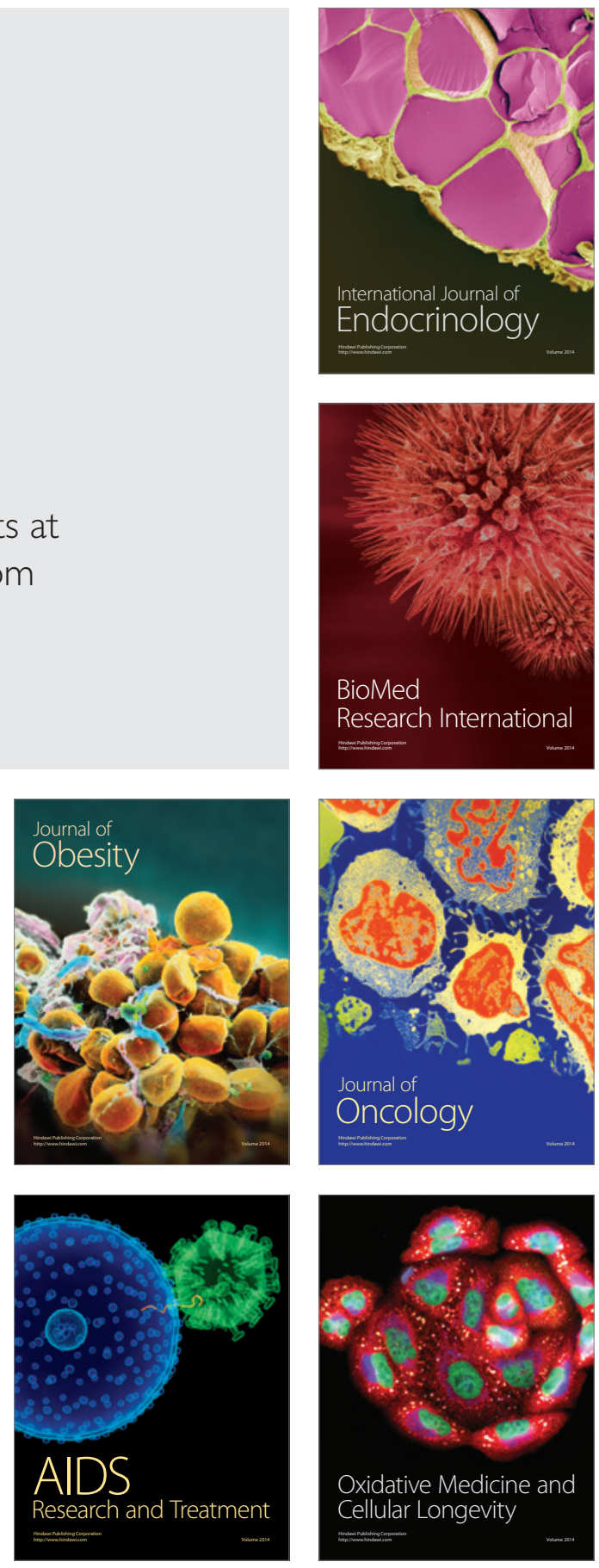